\title{
Quantitative imaging of the clearance systems in the eye and the brain
}

\author{
Wenyu Deng ${ }^{1}$, Crystal Liu ${ }^{1}$, Carlos Parra ${ }^{1}$, Jeffrey R. Sims ${ }^{1}$, Muneeb A. Faiq ${ }^{1}$, Anoop Sainulabdeen ${ }^{1}$, \\ Hana Song ${ }^{1}$, Kevin C. Chan ${ }^{1,2,3,4}$ \\ ${ }^{1}$ Department of Ophthalmology, ${ }^{2}$ Department of Radiology, ${ }^{3}$ Neuroscience Institute, New York University (NYU) School of Medicine, NYU \\ Langone Health, New York, NY, USA; ${ }^{4}$ Center for Neural Science, Faculty of Arts and Science, New York University, New York, NY, USA \\ Correspondence to: Kevin C. Chan, PhD. Departments of Ophthalmology and Radiology, New York University (NYU) School of Medicine, NYU \\ Langone Health, 222 East 41st Street, Room 460, New York, NY 10017, USA. Email: chuenwing.chan@fulbrightmail.org.
}

Submitted Nov 01, 2019. Accepted for publication Nov 26, 2019.

doi: 10.21037/qims.2019.11.18

View this article at: http://dx.doi.org/10.21037/qims.2019.11.18

\section{Introduction}

Debilitating neurodegenerative conditions, such as multiple sclerosis, Alzheimer's and Parkinson's disease, are often presented with the accumulation of metabolic byproducts in brain tissues. Recent studies also suggest linkages between ocular and cerebral diseases, yet the underlying mechanisms remain unclear (1-5). Unlike the rest of the body, the central nervous system (CNS) does not comprise lymphatic vasculature for metabolic waste removal. Instead, several hypotheses have been proposed that rely on the complex but highly regulated clearance mechanisms responsible for an adequate neuronal environment and fluid homeostasis. Understanding the mechanisms of clearance systems in the eye and the brain can help exploit fluid transport and potentially offer new targets for therapy to the visual system and beyond. In this editorial, we describe and criticize how quantitative imaging can play a role in evaluating different models of clearance systems.

\section{Models of the clearance systems in the eye and the brain}

\section{Essential components for biofluid transport in the CNS}

Nutrients and waste products in the brain can be transported through three major fluid compartments: cerebrospinal fluid (CSF) within the subarachnoid space (SAS), interstitial fluid (ISF) within the brain parenchyma, and blood within cerebral vessels. The CSF, produced by choroid plexus, plays a major role in CNS nutrient transport and clearance of waste products, including amyloid plaques and hyperphosphorylated $\tau$-proteins. Failure of such transport and clearance is thought to lead to waste accumulation and toxicity in several neurodegenerative diseases (6-8). The ISF surrounds neurons and glial cells, and takes part in collecting cellular waste products. Blood circulates through cerebral blood vessels. These vessels penetrate the pia mater in the innermost membrane around the brain but remain separate from the brain parenchyma by the glia limitans. The glia limitans are membranes composed of astrocytic endfeets enveloping cerebral vessels. Exchanges between these three components are essential for brain waste clearance and are the subject of active research.

Within the eye, the aqueous humor is secreted into the posterior chamber by non-pigmented ciliary epithelial cells (9). It resembles CSF surrounding the brain and optic nerves with comparable physiologic pressures, production, and drainage dynamics. Its passage through the pupil into the anterior chamber requires downstream outflow mechanisms-the trabecular meshwork and uveoscleral pathways-to maintain optimum intraocular pressure. The corneoscleral and juxtacanalicular tissue layers of the trabecular meshwork drain aqueous humor into the Schlemm's canal, providing access to the episcleral venous system. In the uveoscleral pathway, aqueous humor flows through the interstitial trabeculae of the ciliary bodies and enters the suprachoroidal space and the retinal and optic nerve parenchyma (10). 
Major models for clearance systems in the CNS

To date, three major models have been hypothesized for the waste clearance system in the brain. These include the glymphatic system (6), the intramural periarterial drainage (IPAD) (11), and the meningeal lymphatics $(12,13)$. Figure 1 illustrates each model and how they may function and interact. In brief, the perivascular space surrounding the cerebral vessels is continuous with the SAS within the glia limitans, facilitating CSF and ISF exchange. As CSF circulates within the ventricles and SAS, it traverses the arteriolar glia limitans via aquaporin-4 (AQP4) channels concentrated on the astrocytic endfeet. In the brain parenchyma, debris and metabolic waste can be transported from the ISF via convective flow into the paravenous space. The function of this glia-dependent perivascular network suggests that the paravenous space acts as the penultimate reservoir for drainage into lymphatic-like vessels, hence the term glymphatic (6). Evidence indicates that flow within this system is driven by arterial pulsation, respiration, gentle vasomotion, and the CSF pressure gradient between the SAS and the para-arterial space (14). An alternative IPAD hypothesis proposes that ISF is cleared through the basement membranes of capillaries and vascular smooth muscle cells in the tunica media of cerebral arterioles $(11,15)$. The drainage of ISF in the deep brain may also be controlled by the integrity of myelination (16).

Outside of the brain parenchyma, recent evidence reveals the existence of lymphatic vessels in the meningeal compartment along the dorsal and basal portions of the skull $(12,13)$. The basal meningeal lymphatic vessels seem more involved with CSF/ISF clearance than dorsal meningeal lymphatic vessels and contain lymphatic valves, zipperlike junctions, and characteristics of both capillary and collecting lymphatic vessels. These vessels are close to the SAS that contains a loose intervening arachnoid barrier (13). Hyperspectral fluorescence imaging also demonstrated the flow of quantum dot tracers from the cisterna magna to the submandibular lymph nodes (17).

The eye and the brain are connected by the optic nerve. It was recently found that the extravascular space of the extraocular visual pathway directly communicates with the SAS in humans (18), while CSF may enter the optic nerve via a glymphatic pathway (19). Within the eyeball, recent findings also suggest the possibility of an "ocular glymphatic system". In the inner nuclear layer of the retina, the retinal glial cells called the Müller cells express AQP4 and, like cerebral astrocytes, may be instrumental in maintaining such retinal fluid homeostasis (20). Impaired ocular drainage in multiple sclerosis manifests as an increase in the volume of inner nuclear layer, suggestive of the clinical relevance of this clearance system (20). Apart from glymphatic system, lymphatic drainage from the eye was also observed (9). Fluorescence imaging following injection of quantum dots into the anterior chamber shows afferent pathways into the submandibular lymph nodes in the ipsilateral neck region $(21,22)$. Furthermore, these studies have shown that ocular lymphatic drainage increases following administration of latanoprost which is a prostaglandin F2 $\alpha$ analog (23).

\section{Imaging techniques for monitoring the clearance systems in the eye and the brain}

The mechanisms involved in waste clearance from the eye and brain remain a matter of debate. Development of quantitative imaging techniques can offer an objective measure to verify the properties of the proposed models. Table 1 summarizes recent protocols for imaging the clearance systems in the brains and the eyes of both humans and experimental animal models. In general, most imaging studies of the clearance system involve administrations of exogenous tracers such as fluorescent nanospheres, quantum dots and radio-labeled human serum albumin (HSA). Such tracers of various molecular weights and ligand properties allow for visualization and characterization of CSF $(6-8,12)$ and aqueous humor dynamics and drainages $(22,36)$. Small-sized tracers can move into the interstitium in the brain, or into the anterior chamber via the blood-aqueous barrier, whereas large-sized tracers often remain confined to paravascular spaces after intracisternal injection, or to the ocular bloodstream after systemic administration $(6,7,36,37)$. Ex vivo approaches such as brain slice fluorescence imaging allow histological verification to depict precisely how and where tracers reach and accumulate by examining the specific types of nearby anatomical structures at high spatial resolution (19). However, these experiments are limited to a single time point per subject and are susceptible to biovariability between individuals when testing time- and dose-dependent effects (37). Therefore, non-invasive techniques are being developed to allow longitudinal monitoring of the dynamics of the clearance systems. Recently, photoacoustic imaging has successfully mapped and quantified lymphatic drainage from the eye to neck lymph nodes by leveraging the high contrast of optical imaging and precise spatial resolution of ultrasound in detecting near-infrared nanoparticles (35). 


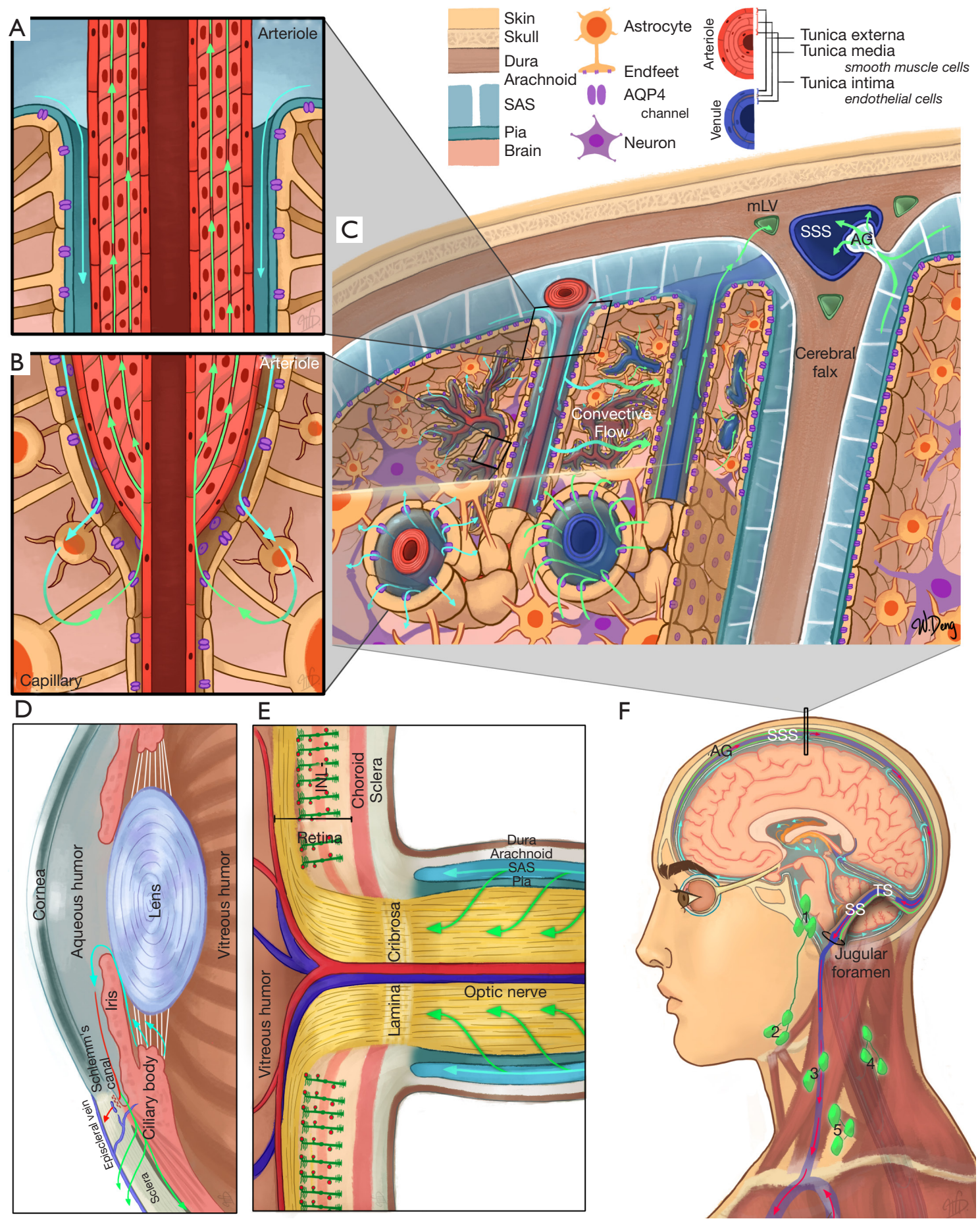

Figure 1 Schematic of major clearance systems in (A,B,C,F) the brain and (D,E,F) the eye. (A,B,C) are representations of the IPAD and glymphatic pathways; (A) is a cross-section of an arteriole and represents CSF flow (cyan arrows) from the SAS into the peri-arterial space, as well as ISF flow (green arrows) through the smooth muscle basement membranes; (B) is a cross-section of an arteriole transitioning into a capillary, where CSF exits the peri-arterial space via AQP4 water channels (purple) located on the astrocytic endfeet before mixing with ISF (cyan green arrow) and entering the smooth muscle basement membranes; (C) is a coronal cross-section through the head and represents the glymphatic pathway, dorsal mLVs, and CSF flow through an AG. CSF flows from the SAS into peri-arterial spaces before flowing into 
the brain parenchyma via AQP4 channels, mixing with ISF, and then entering the perivenous space for drainage via a convective flow. Fluid from the SAS can then drain into the mLVs (green openings) surrounding the SSS; (D) represents a cross-section of the anterior chamber of the eye. Cyan arrows represent production and flow of aqueous humor from the ciliary body. Red arrow represents the trabecular meshwork pathway where aqueous humor flows into the episcleral vein by passing through the Schlemm's canal (blue opening). Green arrow represents the uveoscleral pathway where aqueous humor flows through the interstitial trabeculae of the ciliary bodies and enters the suprachoroidal space; (E) represents a cross-section of the optic nerve head. Müller cells within the retina, which share similar functions to astrocytes in the brain, are represented in dark green, with appendages that wrap around retinal capillaries (red dots), constituting part of the bloodretinal barrier. The broad stripe behind the Müller cells represents the INL. The optic nerve is surrounded by SAS through which CSF flows into the optic nerve; $(\mathrm{F})$ is a diagram of CSF flow within the SAS, originating from the choroid plexus (orange) within the ventricles, as well as dorsal and basal mLVs. These mLVs travelling alongside the TS and SS, exiting out of the jugular foramen with the internal jugular vein, and draining into the deep cervical lymph nodes. Preauricular [1], submandibular [2], superficial cervical [3], deep cervical [4], and supraclavicular [5] lymph nodes are illustrated. IPAD, intramural periarterial drainage; CSF, cerebrospinal fluid; SAS, subarachnoid space; ISF, interstitial fluid; AQP4, aquaporin-4; mLVs, meningeal lymphatic vessels; AG, arachnoid granulation; SSS, superior sagittal sinus; INL, inner nuclear layer; TS, transverse sinuses; SS, sigmoid sinuses.

Hyperspectral fluorescence imaging can track the posterior brain metabolic clearance into dural sinuses associated with lymphatic vessels, as well as the flow of quantum dot tracers from the cisterna magna to the submandibular lymph nodes over time (38). These methods also help characterize waste clearance mechanisms in the eye $(21,23)$.

Although the above tracer-tracking techniques can produce detailed images of CSF and aqueous humor flows in vivo, such images are often restricted to the surfaces of the brain, ocular and body tissues due to limited penetration depth for optical and ultrasound imaging. The majority of their use is also restricted to animal models due to the safety of exogenous materials, and surgical requirements such as invasive injection procedures or the need of a cranial window for a clear optical axis in case of two-photon microscopy. There exists a need of a non-invasive imaging modality that allows translational research of the clearance systems between humans and experimental animal models. Magnetic resonance imaging (MRI) is non-invasive with no depth limitation and may serve this purpose. MRI exploits the magnetic properties of water in biological tissues and allows dynamic monitoring of the clearance systems using paramagnetic contrast agents. For example, gadoliniumbased contrast agents (GBCAs) are passive contrast agents that enhance the T1-weighted MRI signal of CSF following intrathecal or intravenous administration. GBCAs escape the bloodstream into the SAS (39) and their interaction with brain parenchyma can be mediated by the glymphatic system (40-42). GBCAs can also mimic aqueous humor components entering the anterior chamber of the eye via the blood-aqueous barrier $(36,43)$. Their pharmacokinetics varies with the choice of contrast agent (i.e., linear or macrocyclic) and heavily depends on renal function for systemic elimination. The contrast selection may depend on the structure of interest; while macrocyclic GBCAs are more stable, linear GBCAs tend to accumulate in the dentate nucleus and globus pallidus after intravenous administration (44) and in the basal ganglia after intrathecal administration (40). These studies also demonstrated that some contrast is transported from the venous perivascular space into the lymphatic system.

Animal model experiments with GBCAs have demonstrated the capacity of MRI to visualize glymphatic function (45). Comparisons between intrathecal infusions of Gd-DTPA (938 kDa) and GadoSpin (200 kDa) confirmed the fluid transport into paravascular spaces and the sizedependent CSF-ISF exchange typical of glymphatic transport (37). Additionally, AQP4 inhibition reduced clearance after intrathecal infusion of GBCAs, suggesting the dependence of contrast clearance on glymphatics and that MRI is a valid method for glymphatic functional imaging (42). While Gd-DTPA is recently discontinued in the US, Gd-DOTA may offer an alternative to glymphatic imaging with no apparent differences in penetration and distribution into the CSF of healthy rats (46). Reports on human intrathecal injection suggest the feasibility of this approach in clinical settings, but safety concerns do remain (47). Recent studies showed abnormally sustained enhancement of T1-weighted signal in brain parenchyma secondary to reduced rates of gadobutrol clearance from the SAS and reduced glymphatic function in patients treated for idiopathic normal pressure hydrocephalus (48).

Apart from exogenous tracers, non-invasive MRI of clearance systems via endogenous contrasts is also 


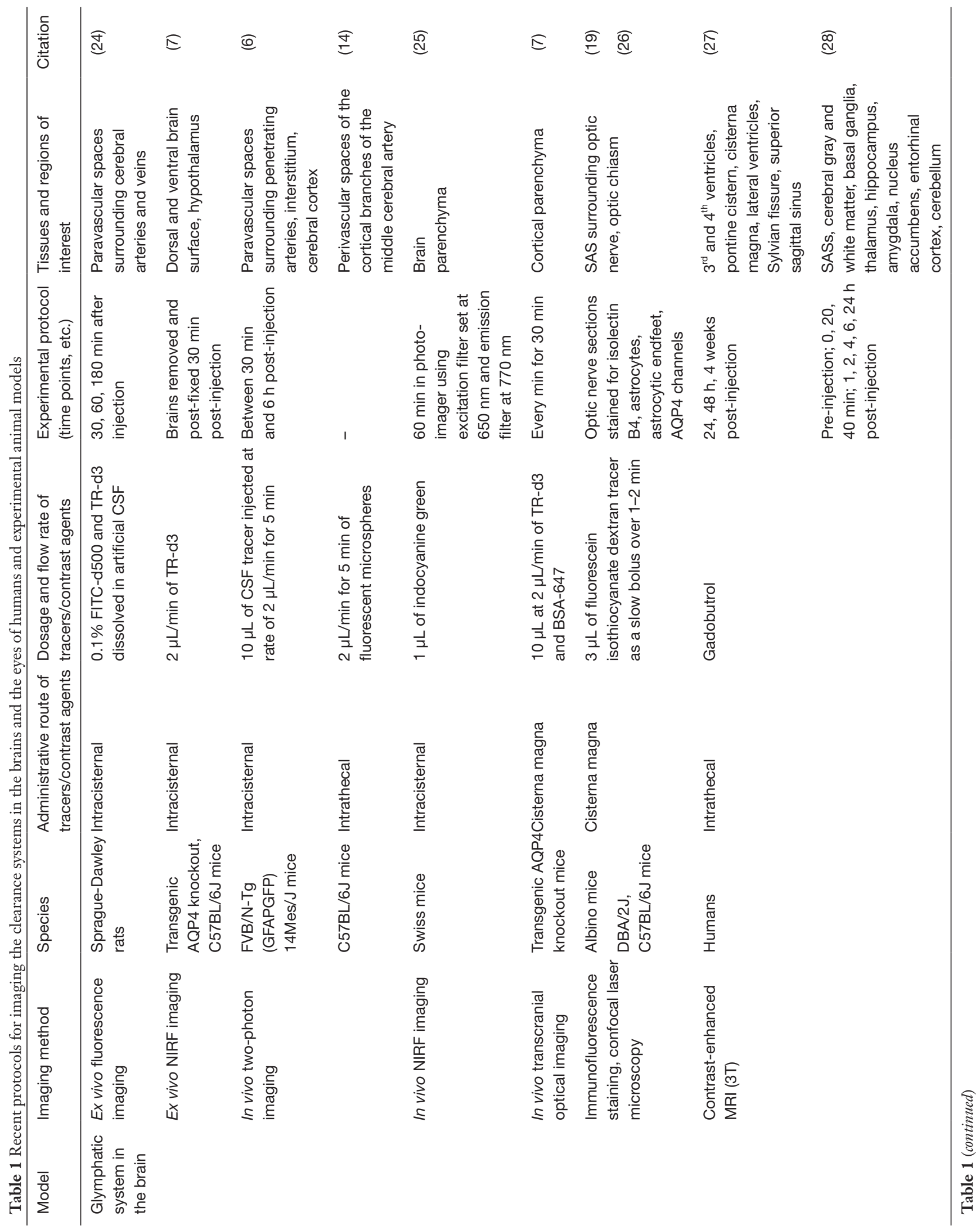




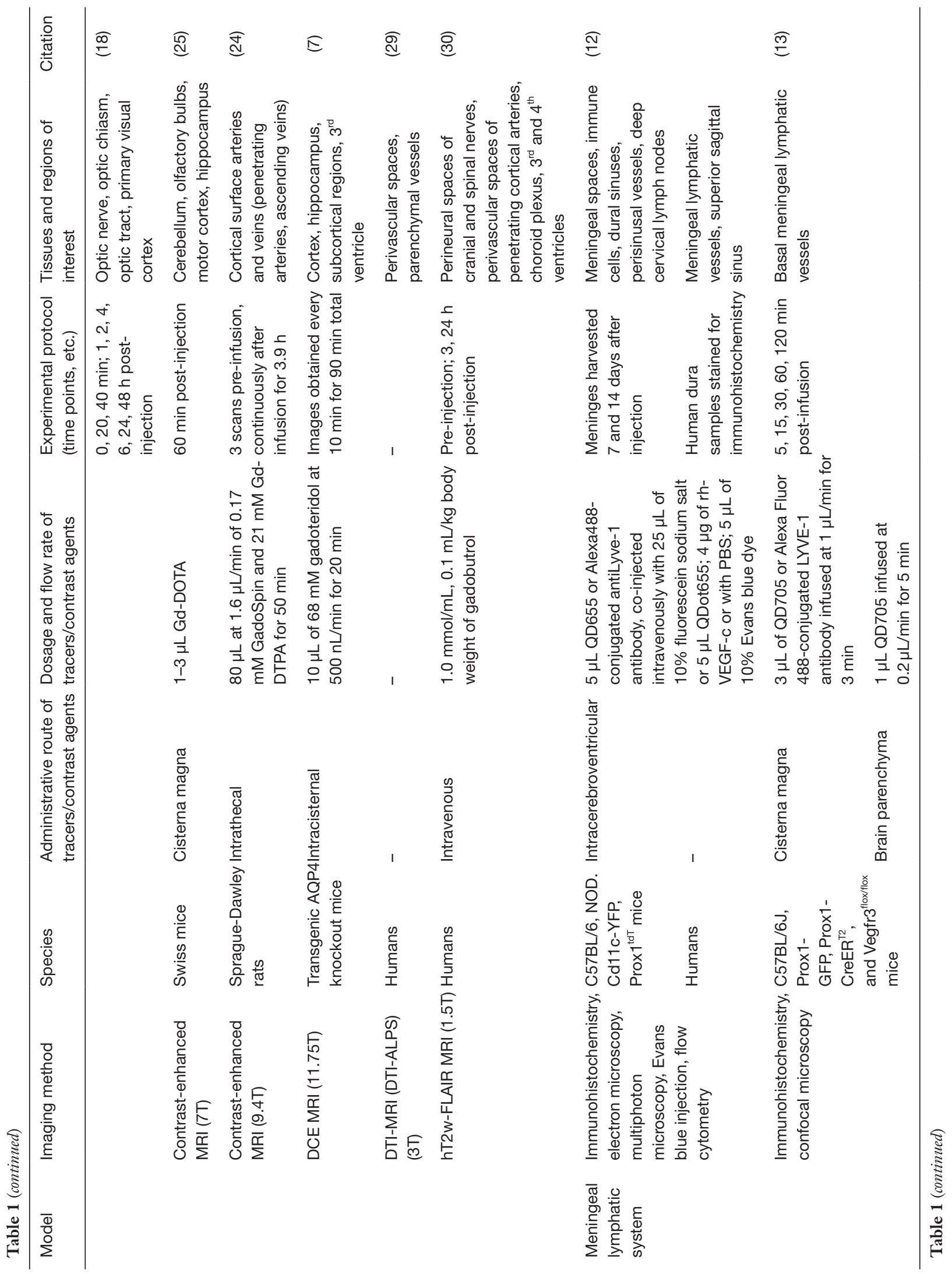




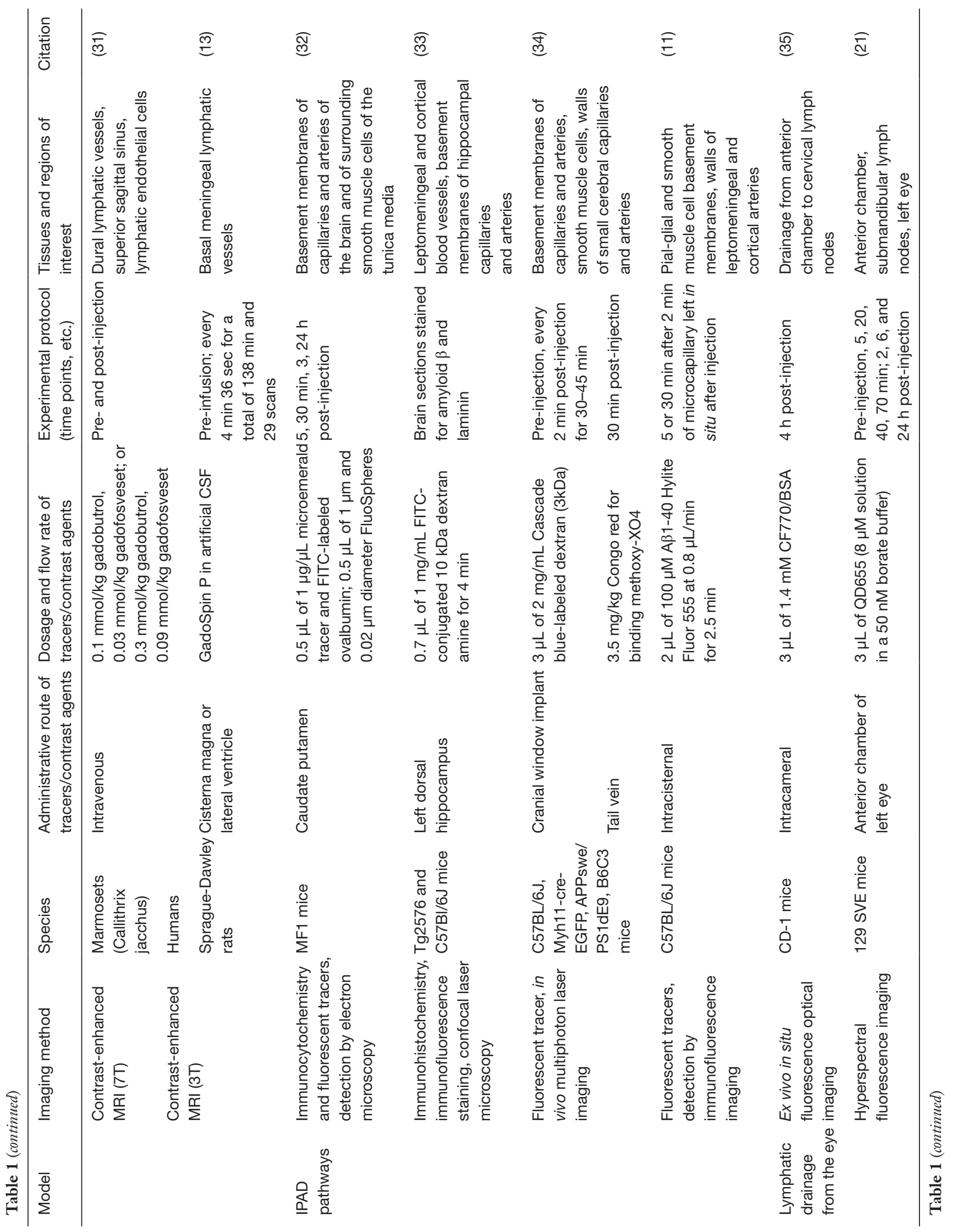




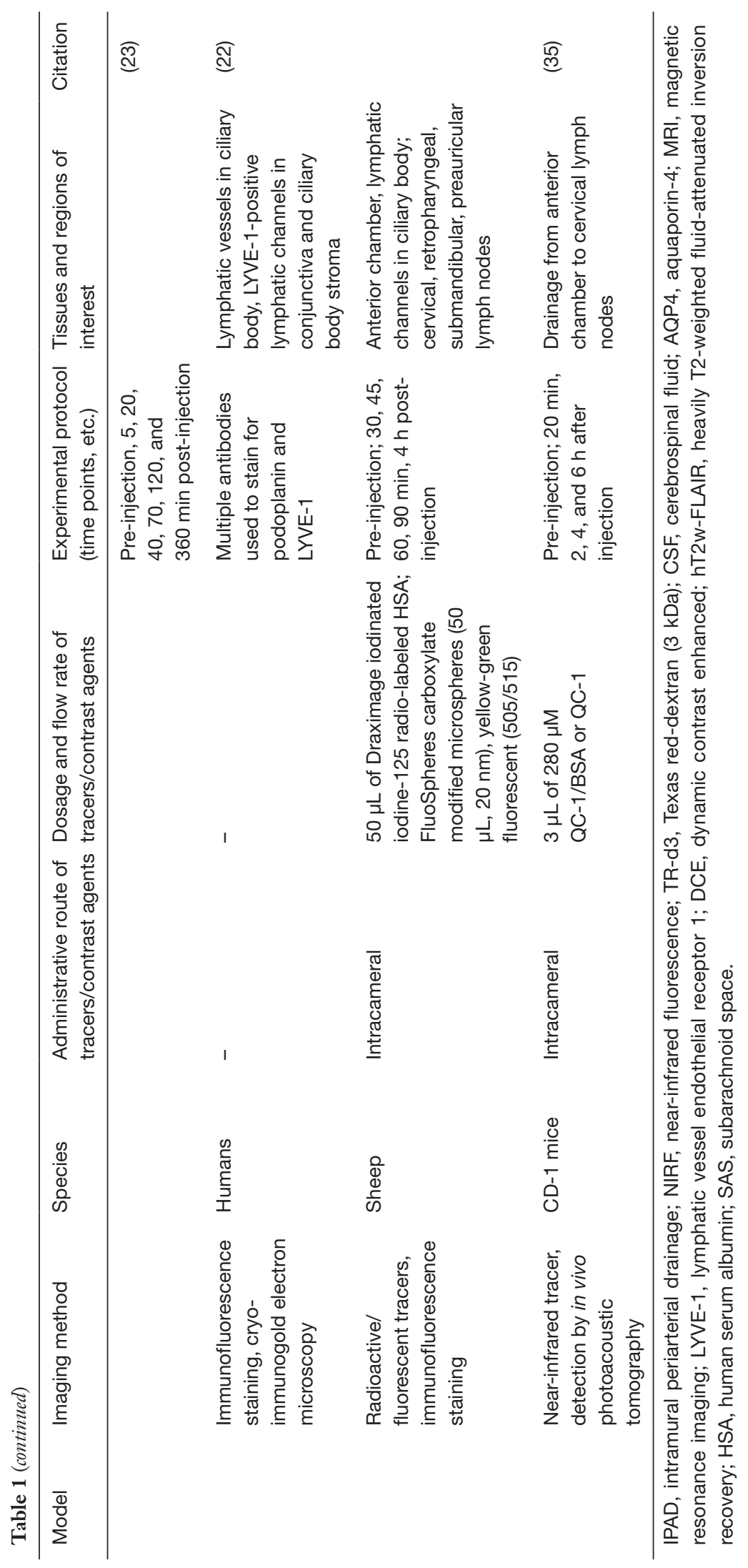


emerging. Several studies have utilized diffusion-weighted MRI techniques for the evaluation of the glymphatic system by detecting intrinsic water movement along the perivascular space $(29,47)$. Research on neurodegenerative diseases has shown significant correlations between mental impairment scores and the perivascular space diffusivity index that is derived from diffusivity patterns in brain projection fibers, association fibers, and the perivascular space (29). Phase-contrast evaluation and magnetization with inversion pulses are also powerful MRI methods for visualizing flow within the CSF space without exogenous material administration (47).

\section{Quantitation of the clearance systems in the eye and the brain}

Quantification of the complete CNS waste clearance system remains challenging partly due to limitations in tracer choices for full eye or brain in vivo research, and in the resolution and specificity of non-invasive imaging techniques. However, indirect measurements in distal locations, synchronization with physiological monitoring and biophysical modeling may help improve the understanding of the relative contributions of individual components to the clearance systems. For example, upon intraocular injection of radioactive tracers, we can quantify the clearance in the trabecular meshwork and uveoscleral pathway separately by measuring the time progression of total radioactivity in the plasma and lymphatic tissues, respectively using a gamma counter (49). Quantification of CSF flow can be accomplished with fluorescent tracers and microspheres infused into the cisterna magna of mice and visualized by two-photon microscopy, while simultaneously measuring pulse and respiration. Microsphere velocity measurements, electrocardiograms, and spectral analyses demonstrate that CSF flow more closely resembles the cardiac cycle than the respiratory cycle, suggesting that the primary driver of CSF flow is perivascular pumping (14). The synchronization of diffusion-weighted MRI acquisition with electrocardiogram also allows for the characterization of the effect of arterial pulsatility on the perivascular space and the surrounding fluid movement in terms of pseudodiffusivity indices (50).

In terms of biophysical modeling, a two-compartment pharmacokinetic model for GBCA-based glymphatic transport has been tested in rats with severe disruption in micro- and macro-vasculature induced by diabetes mellitus (50). The contrast concentration in two compartments (free and bound fractions) is represented by a system of differential equations describing contrast dynamics among the arterial perivasculature and brain parenchyma. The model solution has a biexponential form whose respective fractions and time constants allow the estimation of contrast retention and loss to the perivascular space. This approach has also been used to demonstrate the dependence of glymphatic clearance on corporal position. Experiments showed reduced overall clearance in rats scanned in a prone position compared to supine or right lateral decubitus positions (51). These results were verified with fluorescence and radiolabeled tracer imaging, showing comparatively greater CSF influx in the supine and right lateral decubitus positions.

The spontaneous rhythmic oscillations of vascular tone in the beds of various tissues, including the cerebral tissues, known as vasomotion, are central to the IPAD model for brain waste clearance. This model encompasses a complex system of equations, representing vasomotioninduced intramural periarterial flow through the poroelastic basement membrane, coupled with the elastic response of the middle cerebral artery in the arterial wall model. This model has been proposed to explain the mechanism of IPAD pathways through the basement membranes (15).

\section{Challenges in imaging the clearance systems of the eye and the brain}

Apart from the physical factors from imaging techniques in probing the mechanisms of clearance systems (30), CNS waste clearance appears to be affected by a number of physiological factors such as wakefulness $(52,53)$, anesthesia regimes $(25,53,54)$, exercise $(55)$, age $(7)$, tracer delivery $(7,56)$, and posture $(51,57)$. CSF tracer influx appears to be suppressed in awake subjects (58), increased after voluntary exercise (55), and increased or decreased in sedated subjects depending on the anesthesia regimes (59). In particular, at a high anesthetic dose such as $3 \%$ isoflurane, general anesthesia may have a negative impact on the intracranial CSF circulation $(25,53)$. This occurs not simply by inducing unconsciousness but also by additional mechanisms including repression of norepinephrine release (54). To minimize the effects of anesthesia on solute transport during imaging, anesthesia with dexmedetomidine and lowdose isoflurane has been proposed if awake imaging is not feasible (54).

CNS waste clearance research often requires surgical procedures for the administration of tracers prior to 
image acquisition. In animal studies, imaging of lymphatic drainage in the eye typically relies on intracameral, intravitreal, or subretinal contrast injection. For imaging brain waste clearance, intrathecal catheterization is desirable over intracranial administration since it eliminates the need for a craniotomy (60). SAS catheterization via the atlanto-occipital membrane allows catheter insertion into the cisterna magna or down to the lower levels of the lumbar spinal cord of rats $(61,62)$. Longitudinal studies in rodents typically rely on lumbar intrathecal catheterization for the infusion of tracers into the CSF $(62,63)$. MRI studies of human glymphatic function also rely on lumbar puncture and intrathecal administration of CSF tracers to ensure brain-wide CSF contrast enhancement and clearance $(27,28)$. Intracerebroventricular tracer injection can also be used, yet cautions should be noted when performing invasive procedures for glymphatic studies, as it is reported that intrastriatal injections suppress glymphatic function (7).

\section{Future perspectives}

Despite several advances, questions remain regarding the properties of cerebral and ocular clearance systems and their mechanisms. For example, AQP4 channels are wateronly channels whereas CSF has components much larger in number and size that cannot effectively pass through AQP4 channels. Explorations based on this premise may uncover new substances, transporters, channels, pumps and mechanisms/routes. Better imaging techniques to determine the physiological integrity of CSF and aqueous compartments may also help identify vulnerable individuals with diseases like dementia (64) and glaucoma (26). Delayed tracer clearance from brain parenchyma suggests that contrast-enhanced imaging could potentially be employed to diagnose and examine neurodegenerative diseases in humans and preclinical models $(8,27,47,65)$. In the future, development of a quantitative imaging model system that guides how to manipulate solute transport in appropriate experimental animal models is imperative to facilitate waste clearance and alleviate disease severity $(57,66-70)$. Although most of the current surgical procedures and contrast agents for clearance system studies cannot be used in humans considering safety and FDA approval, quantitative GBCAenhanced MRI has recently been employed to study cerebral and ocular clearance in certain human populations $(18,27,47,71,72)$, whereas diffusion-weighted MRI and phase-contrast MRI could provide information of solute transport without the need of exogenous contrast agents, which could be a major direction for future human imaging research (47). Finally, a unifying model is required to account for the various findings that have been reported so far $(11,73,74)$. Understanding the clearance systems may provide insight into the development of neurological pathologies, ultimately paving the way for neurotherapeutics.

\section{Acknowledgments}

Funding: We thank all collaborators who contributed to our research papers upon which the present commentary is based. This work was supported in part by the National Institutes of Health R01-EY028125, (Bethesda, MD, USA); BrightFocus Foundation G2019103 (Clarksburg, MD, USA); Research to Prevent Blindness/Stavros Niarchos Foundation International Research Collaborators Award (New York, New York); and an unrestricted grant from Research to Prevent Blindness to NYU Langone Health Department of Ophthalmology (New York, NY, USA).

\section{Footnote}

Conflicts of Interest: The authors have no conflicts of interest to declare.

\section{References}

1. Nucci C, Martucci A, Cesareo M, Garaci F, Morrone LA, Russo R, Corasaniti MT, Bagetta G, Mancino R. Links among glaucoma, neurodegenerative, and vascular diseases of the central nervous system. Prog Brain Res 2015;221:49-65.

2. Gupta V, Gupta VB, Chitranshi N, Gangoda S, Vander Wall R, Abbasi M, Golzan M, Dheer Y, Shah T, Avolio A, Chung R, Martins R, Graham S. One protein, multiple pathologies: multifaceted involvement of amyloid beta in neurodegenerative disorders of the brain and retina. Cell Mol Life Sci 2016;73:4279-97.

3. Faiq MA, Wollstein G, Schuman JS, Chan KC. Cholinergic nervous system and glaucoma: From basic science to clinical applications. Prog Retin Eye Res 2019;72:100767.

4. Wostyn P, De Groot V, Van Dam D, Audenaert K, Killer HE, De Deyn PP. Age-related macular degeneration, glaucoma and Alzheimer's disease: amyloidogenic diseases with the same glymphatic background? Cell Mol Life Sci 2016;73:4299-301.

5. Trivedi V, Bang JW, Parra C, Colbert MK, O'Connell C, 
Arshad A, Faiq MA, Conner IP, Redfern MS, Wollstein G, Schuman JS, Cham R, Chan KC. Widespread brain reorganization perturbs visuomotor coordination in early glaucoma. Sci Rep 2019;9:14168.

6. Iliff JJ, Wang M, Liao Y, Plogg BA, Peng W, Gundersen GA, Benveniste H, Vates GE, Deane R, Goldman SA, Nagelhus EA, Nedergaard M. A paravascular pathway facilitates CSF flow through the brain parenchyma and the clearance of interstitial solutes, including amyloid . Sci Transl Med 2012;4:147ra111.

7. Mestre H, Hablitz LM, Xavier AL, Feng W, Zou W, Pu T, Monai H, Murlidharan G, Castellanos Rivera RM, Simon MJ, Pike MM, Plá V, Du T, Kress BT, Wang X, Plog BA, Thrane AS, Lundgaard I, Abe Y, Yasui M, Thomas JH, Xiao M, Hirase H, Asokan A, Iliff JJ, Nedergaard M. Aquaporin-4-dependent glymphatic solute transport in the rodent brain. Elife 2018. doi: 10.7554/eLife.40070.

8. Tarasoff-Conway JM, Carare RO, Osorio RS, Glodzik L, Butler T, Fieremans E, Axel L, Rusinek H, Nicholson C, Zlokovic BV, Frangione B, Blennow K, Menard J, Zetterberg H, Wisniewski T, de Leon MJ. Clearance systems in the brain-implications for Alzheimer disease. Nat Rev Neurol 2015;11:457-70.

9. Yucel Y, Gupta N. Lymphatic drainage from the eye: a new target for therapy. Prog Brain Res 2015;220:185-98.

10. Bill A. Aqueous humor dynamics in monkeys (Macaca irus and Cercopithecus ethiops). Exp Eye Res 1971;11:195-206.

11. Albargothy NJ, Johnston DA, MacGregor-Sharp M, Weller RO, Verma A, Hawkes CA, Carare RO. Convective influx/glymphatic system: tracers injected into the CSF enter and leave the brain along separate periarterial basement membrane pathways. Acta Neuropathol 2018;136:139-52.

12. Louveau A, Smirnov I, Keyes TJ, Eccles JD, Rouhani SJ, Peske JD, Derecki NC, Castle D, Mandell JW, Lee KS, Harris TH, Kipnis J. Structural and functional features of central nervous system lymphatic vessels. Nature 2015;523:337-41.

13. Ahn JH, Cho H, Kim JH, Kim SH, Ham JS, Park I, Suh SH, Hong SP, Song JH, Hong YK, Jeong Y, Park SH, Koh GY. Meningeal lymphatic vessels at the skull base drain cerebrospinal fluid. Nature 2019;572:62-6.

14. Mestre H, Tithof J, Du T, Song W, Peng W, Sweeney AM, Olveda G, Thomas JH, Nedergaard M, Kelley DH. Flow of cerebrospinal fluid is driven by arterial pulsations and is reduced in hypertension. Nat Commun 2018;9:4878.

15. Aldea R, Weller RO, Wilcock DM, Carare RO,
Richardson G. Cerebrovascular smooth muscle cells as the drivers of intramural periarterial drainage of the brain. Front Aging Neurosci 2019;11:1.

16. Wang A, Wang R, Cui D, Huang X, Yuan L, Liu H, Fu Y, Liang L, Wang W, He Q, Shi C, Guan X, Teng Z, Zhao G, Li Y, Gao Y, Han H. The drainage of interstitial fluid in the deep brain is controlled by the integrity of myelination. Aging Dis 2019;10:937-48.

17. Mathieu E, Gupta N, Macdonald RL, Ai J, Yücel YH. In vivo imaging of lymphatic drainage of cerebrospinal fluid in mouse. Fluids Barriers CNS 2013;10:35.

18. Jacobsen HH, Ringstad G, Jorstad OK, Moe MC, Sandell T, Eide PK. The human visual pathway communicates directly with the subarachnoid space. Invest Ophthalmol Vis Sci 2019;60:2773-80.

19. Mathieu E, Gupta N, Ahari A, Zhou X, Hanna J, Yücel $\mathrm{YH}$. Evidence for cerebrospinal fluid entry into the optic nerve via a glymphatic pathway. Invest Ophthalmol Vis Sci 2017;58:4784-91.

20. Petzold A. Retinal glymphatic system: an explanation for transient retinal layer volume changes? Brain 2016;139:2816-9.

21. Tam AL, Gupta N, Zhang Z, Yücel YH. Quantum dots trace lymphatic drainage from the mouse eye. Nanotechnology 2011;22:425101.

22. Yücel YH, Johnston MG, Ly T, Patel M, Drake B, Gümü E, Fraenkl SA, Moore S, Tobbia D, Armstrong D, Horvath E, Gupta N. Identification of lymphatics in the ciliary body of the human eye: a novel "uveolymphatic" outflow pathway. Exp Eye Res 2009;89:810-9.

23. Tam AL, Gupta N, Zhang Z, Yücel YH. Latanoprost stimulates ocular lymphatic drainage: an in vivo nanotracer study. Transl Vis Sci Technol 2013;2:3.

24. Iliff JJ, Wang M, Zeppenfeld DM, Venkataraman A, Plog BA, Liao Y, Deane R, Nedergaard M. Cerebral arterial pulsation drives paravascular CSF-interstitial fluid exchange in the murine brain. J Neurosci 2013;33:18190-9.

25. Gakuba C, Gaberel T, Goursaud S, Bourges J, Di Palma C, Quenault A, Martinez de Lizarrondo S, Vivien D, Gauberti M. General anesthesia inhibits the activity of the "glymphatic system". Theranostics 2018;8:710-22.

26. Mathieu E, Gupta N, Paczka-Giorgi LA, Zhou X, Ahari A, Lani R, Hanna J, Yücel YH. Reduced cerebrospinal fluid inflow to the optic nerve in glaucoma. Invest Ophthalmol Vis Sci 2018;59:5876-84.

27. Ringstad G, Vatnehol SAS, Eide PK. Glymphatic MRI in idiopathic normal pressure hydrocephalus. Brain 2017;140:2691-705. 
28. Ringstad G, Valnes LM, Dale AM, Pripp AH, Vatnehol SS, Emblem KE, Mardal KA, Eide PK. Brain-wide glymphatic enhancement and clearance in humans assessed with MRI. JCI Insight 2018. doi: 10.1172/jci.insight.121537.

29. Taoka T, Masutani Y, Kawai H, Nakane T, Matsuoka K, Yasuno F, Kishimoto T, Naganawa S. Evaluation of glymphatic system activity with the diffusion MR technique: diffusion tensor image analysis along the perivascular space (DTI-ALPS) in Alzheimer's disease cases. Jpn J Radiol 2017;35:172-8.

30. Deike-Hofmann K, Reuter J, Haase R, Paech D, Gnirs R, Bickelhaupt S, Forsting M, Heussel CP, Schlemmer HP, Radbruch A. Glymphatic pathway of gadoliniumbased contrast agents through the brain: overlooked and misinterpreted. Invest Radiol 2019;54:229-37.

31. Absinta M, Ha SK, Nair G, Sati P, Luciano NJ, Palisoc M, Louveau A, Zaghloul KA, Pittaluga S, Kipnis J, Reich DS. Human and nonhuman primate meninges harbor lymphatic vessels that can be visualized noninvasively by MRI. Elife 2017. doi: 10.7554/eLife.29738.

32. Carare RO, Bernardes-Silva M, Newman TA, Page AM, Nicoll JA, Perry VH, Weller RO. Solutes, but not cells, drain from the brain parenchyma along basement membranes of capillaries and arteries: significance for cerebral amyloid angiopathy and neuroimmunology. Neuropathol Appl Neurobiol 2008;34:131-44.

33. Hawkes CA, Hartig W, Kacza J, Schliebs R, Weller RO, Nicoll JA, Carare RO. Perivascular drainage of solutes is impaired in the ageing mouse brain and in the presence of cerebral amyloid angiopathy. Acta Neuropathol 2011;121:431-43.

34. Arbel-Ornath M, Hudry E, Eikermann-Haerter K, Hou S, Gregory JL, Zhao L, Betensky RA, Frosch MP, Greenberg SM, Bacskai BJ. Interstitial fluid drainage is impaired in ischemic stroke and Alzheimer's disease mouse models. Acta Neuropathol 2013;126:353-64.

35. Yücel YH, Cardinell K, Khattak S, Zhou X, Lapinski M, Cheng F, Gupta N. Active lymphatic drainage from the eye measured by noninvasive photoacoustic imaging of near-infrared nanoparticles. Invest Ophthalmol Vis Sci 2018;59:2699-707.

36. Ho LC, Conner IP, Do CW, Kim SG, Wu EX, Wollstein $\mathrm{G}$, Schuman JS, Chan KC. In vivo assessment of aqueous humor dynamics upon chronic ocular hypertension and hypotensive drug treatment using gadolinium-enhanced MRI. Invest Ophthalmol Vis Sci 2014;55:3747-57.

37. Iliff JJ, Lee H, Yu M, Feng T, Logan J, Nedergaard M, Benveniste H. Brain-wide pathway for waste clearance captured by contrast-enhanced MRI. J Clin Invest 2013;123:1299-309.

38. Iliff JJ, Goldman SA, Nedergaard M. Implications of the discovery of brain lymphatic pathways. Lancet Neurol 2015;14:977-9.

39. Naganawa S, Yamazaki M, Kawai H, Sone M, Nakashima T. Contrast enhancement of the anterior eye segment and subarachnoid space: detection in the normal state by heavily T2-weighted 3D FLAIR. Magn Reson Med Sci 2011;10:193-9.

40. Öner AY, Barutcu B, Aykol , Tali ET. Intrathecal contrastenhanced magnetic resonance imaging-related brain signal changes: residual gadolinium deposition? Invest Radiol 2017;52:195-7.

41. Taoka T, Naganawa S. Gadolinium-based contrast media, cerebrospinal fluid and the glymphatic system: possible mechanisms for the deposition of gadolinium in the brain. Magn Reson Med Sci 2018;17:111-9.

42. Harrison IF, Siow B, Akilo AB, Evans PG, Ismail O, Ohene Y, Nahavandi P, Thomas DL, Lythgoe MF, Wells JA. Non-invasive imaging of CSF-mediated brain clearance pathways via assessment of perivascular fluid movement with diffusion tensor MRI. Elife 2018. doi: 10.7554/eLife.34028.

43. Chan KC, Fu QL, Guo H, So KF, Wu EX. GD-DTPA enhanced MRI of ocular transport in a rat model of chronic glaucoma. Exp Eye Res 2008;87:334-41.

44. Kanda T, Ishii K, Kawaguchi H, Kitajima K, Takenaka D. High signal intensity in the dentate nucleus and globus pallidus on unenhanced T1-weighted MR images: relationship with increasing cumulative dose of a gadolinium-based contrast material. Radiology 2014;270:834-41.

45. Ding G, Chopp M, Li L, Zhang L, Davoodi-Bojd E, Li Q, Zhang Z, Jiang Q. MRI investigation of glymphatic responses to Gd-DTPA infusion rates. J Neurosci Res 2018;96:1876-86.

46. Jost G, Frenzel T, Lohrke J, Lenhard DC, Naganawa S, Pietsch H. Penetration and distribution of gadoliniumbased contrast agents into the cerebrospinal fluid in healthy rats: a potential pathway of entry into the brain tissue. Eur Radiol 2017;27:2877-85.

47. Taoka T, Naganawa S. Glymphatic imaging using MRI. J Magn Reson Imaging 2020;51:11-24.

48. Eide PK, Ringstad G. Delayed clearance of cerebrospinal fluid tracer from entorhinal cortex in idiopathic normal pressure hydrocephalus: a glymphatic magnetic resonance imaging study. J Cereb Blood Flow 
Metab 2019;39:1355-68.

49. Kim M, Johnston MG, Gupta N, Moore S, Yücel YH. A model to measure lymphatic drainage from the eye. Exp Eye Res 2011;93:586-91.

50. Davoodi-Bojd E, Ding G, Zhang L, Li Q, Li L, Chopp M, Zhang Z, Jiang Q. Modeling glymphatic system of the brain using MRI. NeuroImage 2019;188:616-27.

51. Lee H, Xie L, Yu M, Kang H, Feng T, Deane R, Logan J, Nedergaard M, Benveniste H. The effect of body posture on brain glymphatic transport. J Neurosci 2015;35:11034-44.

52. Fultz NE, Bonmassar G, Setsompop K, Stickgold RA, Rosen BR, Polimeni JR, Lewis LD. Coupled electrophysiological, hemodynamic, and cerebrospinal fluid oscillations in human sleep. Science 2019;366:628-31.

53. Benveniste H, Heerdt PM, Fontes M, Rothman DL, Volkow ND. Glymphatic system function in relation to anesthesia and sleep states. Anesth Analg 2019;128:747-58.

54. Benveniste H, Lee H, Ding F, Sun Q, Al-Bizri E, Makaryus R, Probst S, Nedergaard M, Stein EA, Lu H. Anesthesia with dexmedetomidine and low-dose isoflurane increases solute transport via the glymphatic pathway in rat brain when compared with high-dose isoflurane. Anesthesiology 2017;127:976-88.

55. von Holstein-Rathlou S, Petersen NC, Nedergaard M. Voluntary running enhances glymphatic influx in awake behaving, young mice. Neurosci Lett 2018;662:253-8.

56. Albargothy NJ, Sharp MM, Gatherer M, Morris A, Weller RO, Hawkes C, Carare RO. Investigating the lymphatic drainage of the brain: essential skills and tools. Methods Mol Biol 2017;1559:343-65.

57. Lundgaard I, Lu ML, Yang E, Peng W, Mestre H, Hitomi E, Deane R, Nedergaard M. Glymphatic clearance controls state-dependent changes in brain lactate concentration. J Cereb Blood Flow Metab 2017;37:2112-24.

58. Xie L, Kang H, Xu Q, Chen MJ, Liao Y, Thiyagarajan M, O'Donnell J, Christensen DJ, Nicholson C, Iliff JJ, Takano T, Deane R, Nedergaard M. Sleep drives metabolite clearance from the adult brain. Science 2013;342:373-7.

59. Hablitz LM, Vinitsky HS, Sun Q, Stæger FF, Sigurdsson B, Mortensen KN, Lilius TO, Nedergaard M. Increased glymphatic influx is correlated with high EEG delta power and low heart rate in mice under anesthesia. Sci Adv 2019;5:eaav5447.

60. Ramos M, Burdon Bechet N, Battistella R, Pavan C, Xavier ALR, Nedergaard M, Lundgaard I. Cisterna magna injection in rats to study glymphatic function. Methods
Mol Biol 2019;1938:97-104.

61. Yaksh TL, Rudy TA. Chronic catheterization of the spinal subarachnoid space. Physiol Behav 1976;17:1031-6.

62. Yang L, Kress BT, Weber HJ, Thiyagarajan M, Wang B, Deane R, Benveniste H, Iliff JJ, Nedergaard M. Evaluating glymphatic pathway function utilizing clinically relevant intrathecal infusion of CSF tracer. J Transl Med 2013;11:107.

63. Wu WP, Xu XJ, Hao JX. Chronic lumbar catheterization of the spinal subarachnoid space in mice. J Neurosci Methods 2004;133:65-9.

64. Verheggen ICM, Van Boxtel MPJ, Verhey FRJ, Jansen JFA, Backes WH. Interaction between blood-brain barrier and glymphatic system in solute clearance. Neurosci Biobehav Rev 2018;90:26-33.

65. Rasmussen MK, Mestre H, Nedergaard M. The glymphatic pathway in neurological disorders. Lancet Neurol 2018;17:1016-24.

66. Plog BA, Mestre H, Olveda GE, Sweeney AM, Kenney HM, Cove A, Dholakia KY, Tithof J, Nevins TD, Lundgaard I, Du T, Kelley DH4, Nedergaard M. Transcranial optical imaging reveals a pathway for optimizing the delivery of immunotherapeutics to the brain. JCI Insight 2018.doi: 10.1172/jci.insight.120922.

67. Huber VJ, Igarashi H, Ueki S, Kwee IL, Nakada T. Aquaporin-4 facilitator TGN-073 promotes interstitial fluid circulation within the blood-brain barrier: $[17 \mathrm{O}] \mathrm{H} 2 \mathrm{O}$ JJVCPE MRI study. Neuroreport 2018;29:697-703.

68. Pirici I, Balsanu TA, Bogdan C, Margaritescu C, Divan T, Vitalie V, Mogoanta L, Pirici D, Carare RO, Muresanu DF. Inhibition of Aquaporin-4 Improves the Outcome of Ischaemic Stroke and Modulates Brain Paravascular Drainage Pathways. Int J Mol Sci 2018;19:46.

69. Kida T, Oku H, Horie T, Fukumoto M, Okuda Y, Morishita S, Ikeda T. Implication of VEGF and aquaporin 4 mediating Muller cell swelling to diabetic retinal edema. Graefes Arch Clin Exp Ophthalmol 2017;255:1149-57.

70. Peng W, Achariyar TM, Li B, Liao Y, Mestre H, Hitomi E, Regan S, Kasper T, Peng S, Ding F, Benveniste H, Nedergaard M, Deane R. Suppression of glymphatic fluid transport in a mouse model of Alzheimer's disease. Neurobiol Dis 2016;93:215-25.

71. Hitomi E, Simpkins AN, Luby M, Latour LL, Leigh RJ, Leigh R. Blood-ocular barrier disruption in patients with acute stroke. Neurology 2018;90:e915-23.

72. Watts R, Steinklein JM, Waldman L, Zhou X, Filippi CG. Measuring glymphatic flow in man using quantitative 
contrast-enhanced MRI. AJNR Am J Neuroradiol 2019;40:648-51.

73. Louveau A, Plog BA, Antila S, Alitalo K, Nedergaard M, Kipnis J. Understanding the functions and relationships of the glymphatic system and meningeal lymphatics. J Clin Invest 2017;127:3210-9.

Cite this article as: Deng W, Liu C, Parra C, Sims JR, Faiq MA, Sainulabdeen A, Song H, Chan KC. Quantitative imaging of the clearance systems in the eye and the brain. Quant Imaging Med Surg 2020;10(1):1-14. doi: 10.21037/qims.2019.11.18
74. Wostyn P, De Groot V, Van Dam D, Audenaert K, Killer HE, De Deyn PP. The glymphatic hypothesis of glaucoma: a unifying concept incorporating vascular, biomechanical, and biochemical aspects of the disease. Biomed Res Int 2017;2017:5123148. 